\title{
Reduction of Papr in Ofdm Systems Using Clipping and Differential Scaling
}

\author{
N.Santosh kumar ${ }^{1}$, P.Kumari ${ }^{2}$, E.Suresh ${ }^{3}$, V.Radha krishna ${ }^{4}$, R.Divya kanthi ${ }^{5}$ \\ ${ }^{l}$ (Electronics and communication engineering, lendi institute of engg and tech) \\ ${ }^{2}$ (Electronics and communication engineering, lendi institute of engg and tech) \\ ${ }^{3}$ (Electronics and communication engineering, lendi institute of engg and tech) \\ ${ }^{4}$ (Electronics and communication engineering, lendi institute of engg and tech) \\ 5 (Electronics and communication engineering ,lendi institute of engg and tech)
}

\begin{abstract}
Orthogonal Frequency Division Multiplexing (OFDM) has grown to a popular communication technique for high speed communication in the last decade. OFDM is an important member of the multicarrier modulation (MC) techniques and it has been adopted as a standard for various high data rate wireless communication systems due to its spectral bandwidth efficiency, robustness to frequency selective fading channels, etc. Although it is having many advantages it has two major drawbacks namely high PAPR and inter carrier interference (ICI). This paper is aimed to reduce the effect of PAPR and we proposed two techniques for the reduction of high Peak to Average Power Ratio (PAPR) based on Clipping, Differential Scaling.

keywords: Orthogonal frequency division multiplexing (OFDM), partial transmit sequences (PTS), peak-toaverage power ratio(PAPR), selected mapping (SLM), tone reservation (TR), inter carrier interference (ICI), Companding, Active constellation, Multi carrier(MC).
\end{abstract}

\section{Introduction}

OFDM evolution [1] can be divided into three parts namely1.Frequency Division Multiplexing (FDM) 2.Multicarrier Communication (MC) 3.Orthogonal Frequency Division Multiplexing

1. FDM is the concept of using different frequency channels to carry the information of different users. Each channel is identified by the central frequency of transmission. To ensure that the signal of one channel does not overlap with the signal from an adjacent one, some gap or guard band is left between different channels.

2. Multicarrier (MC) is actually the concept of splitting a signal into a number of signals, modulating each of these new signals over its own frequency channels and multiplexing these different frequency channels together in an FDM manner.

3. OFDM is derived from the concept of MC where the different carriers are orthogonal to each other. Orthogonal in this respect means that the signals are totally independent. It is achieved by ensuring that the carriers are placed exactly at the nulls in the modulation spectra of each other.

OFDM is simply defined as a form of multi-carrier modulation where the carrier spacing is carefully selected so that each sub carrier is orthogonal to the other sub carriers. Two signals are orthogonal if their dot product is zero. that is, if you take two signals multiply them together and if their integral over an interval is zero, then two signals are orthogonal in that interval. (This is also a base for fourier series where we are going to find a set of co sinusoidal signals components present in a signal).

Orthogonality can be achieved by carefully selecting carrier spacing, such as letting the carrier spacing be equal to the reciprocal of the useful symbol period. As the sub carriers are orthogonal, the spectrum of each carrier has a null at the center frequency of each of the other carriers in the system.

(OFDM) constitutes a promising technology for high speed transmission in frequency selective fading environments[2] and it presents several important advantages, some of which are High spectral efficiency, Simple implementation (with IDFT/DFT pairs), Mitigation of Inter symbol interference (ISI) and robustness to frequency selective fading Environments. This is a promising modulation technique that is increasingly being considered for adoption by $4 \mathrm{G}$ community. It is a potential candidate for multimedia Wireless services that require high bit rates over mobile radio channels.

\section{Draw Backs Of Ofdm}

Although we are having many advantages of OFDM It has two major limitations of OFDM systems are Peak-to-Average Power Ratio (PAPR) and Inter Carrier Interference (ICI). PAPR is a measurement of waveform calculated from the peak amplitude of the waveform divided by the RMS value of the waveform and this large peak occurs due to the constructive superimposition with a number of subcarriers or the summation of 
a large number of subcarriers. This high PAPR demands high power amplifiers (HPA) at the transmitter. The non-linearity effects of HPA on the transmitted OFDM symbols are spectral spreading, inter modulation and changing the signal constellation. In other words, the non-linear distortion causes both in band and out-of band interference to signals. coming to ICI in this case OFDM system is vulnerable to frequency-offset errors between the transmitted \& received signals, which may be caused by Doppler shift in the channel or by difference between the transmitter and receiver local oscillator frequencies. Hence orthogonality is lost between subcarriers resulting in inter carrier interference (ICI). If ICI in not properly compensated it results in power leakage among the subcarriers and orthogonality between them will be lost.

\section{Peak To Average Power Ratio}

The frequency-domain symbols of an OFDM frame is denoted by $\mathbf{X}=[X(0), X(1), \ldots, X(N c-1)] T$, where $N c$ is the number of subcarriers. It is assumed that $X(k) € C$, where $C$ is the set of constellation points. The vector $\mathbf{x}=[x(0), x(1), \ldots, x(N-1)] T$ contains the time-domain samples of the complex baseband OFDM signal as given by

$$
x(n)=1 / \sqrt{ } N \sum_{k=0}^{N c-1} X(\mathrm{k}) e^{j 2 \pi n k / N}
$$

where $j=\sqrt{ }-1$, and $N / N c$ is the oversampling ratio. It is clear that $\mathbf{x}=\operatorname{IFFTN}\{\mathbf{X}\}$, where $\operatorname{IFFTN}\{\}$ is the $N$-point inverse fast Fourier transform (IFFT) operation. where $\mathbf{x}$ represents the time domain symbols. And $\mathbf{X}$ represents the frequency domain symbols According to above equations, the time-domain samples are the sum of $N c$ independent terms. When $N c$ is large, based on the central limit theorem, the time-domain samples have a Gaussian distribution; thus, they may have large amplitudes[10] which leads to high PAPR. To overcome this problem, some techniques have been proposed in this paper.

The PAPR of the OFDM frame is defined by

$$
\operatorname{PAPR}(\mathrm{x})=\frac{\max _{n}\left\{|x(n)|^{2}\right\}}{E\left\{|x(n)|^{2}\right\}}
$$

where $E\{$.$\} is the mathematical expectation$

$x(n)$ is the time domain signal

\section{Effects Of High Papr}

The signal reveals vulnerable to NONLINEAR distortions induced by the high power amplifier (HPA) of the transmitter which leads to SIGNAL-TO-NOISE RATIO degradation.

The high peaks of the OFDM signal due to high PAPR demand enough dynamic range of DACs. For large dynamic range with a given sampling rate if we use low precision DACs they result in high quantization noise causing high BER.

The large PAPR also results in the increased complexity of analog-to-digital converter (ADC) and digital-to-analog converter (DAC)[15].

High PAPR at the transmitter, which causes signal distortion such as in-band distortion and out-of band radiation due to the nonlinearity of the high power amplifier (HPA) .The large variation in signal amplitudes also affects the HPA.

The non linear characteristics of HPA results in inter-modulation between the different sub carriers and leads to an increase in bit error rate (BER)[14].

So the HPA requires a large back off from the PEAK POWER to reduce the distortion caused by the nonlinearity of HPA and this gives rise to a low power efficiency, which is a significant burden, especially in mobile terminals[15]

To overcome above problems we require costly hardware and complex systems. Therefore to lessen the difficulty of complex hardware design it has become imperative to employ efficient PAPR reduction techniques.

\section{Techniques To Reduce High Papr}

Numerous schemes have been proposed to solve the PAPR problem but no specific PAPR reduction scheme can be considered as the best solution. Since the criteria involve trade-offs like complexity, computational time, memory requirements, data rate loss and high distortion.

Therefore it is needed to compromise the criteria to meet the system requirements. The classical approaches to eliminate this problem are Clipping[3] and filtering, companding[4] Channel coding, Frame superposition using tone reservation(TR) [5],[6], block coding [11], [12], and active constellation extension 
[13],Probabilistic solutions (selected mapping (SLM)[7],[8] and interleaving are well-known probabilistic methods.)

\section{Proposed Tecniques}

Here in this paper we propose two techniques 1.clipping 2.Differential scaling. In the $1^{\text {st }}$ technique Clipping is a way of reducing PAPR by simply limiting the maximum amplitude of the OFDM signal, such that all signal values are limited to the threshold. Clipping the OFDM signal before amplification is a simple method to limit PAPR.

In the second technique differential scaling differential scaling the peak value of the signal is scaled to higher and lower values than the original peak value to decrease the high PAPR and we also proposed scale up, scale down, scale up down and different values for alpha and beta are taken.

\section{Ofdmsystem}

The incoming data bits are mapped onto the constellation plane for the corresponding M-PSK or MQAM scheme and complex symbols are generated. These symbols are to be transmitted independently on to the subcarriers. To achieve this, they are fed parallel to the input of the N-point IFFT. They represent the frequency domain data set. Inverse Fourier transform converts this frequency domain data set into its corresponding time domain representation. Specifically, IFFT is useful for OFDM because it generates samples of waveforms with orthogonal frequency components. PAPR reduction is applied at the output of the IFFT block and the OFDM symbols are then transmitted over the channel with energy per bit as $E b$. The channel considered here is an Additive White Gaussian Noise (AWGN) channel with mean zero and variance No. At the receiver the inverse PAPR reduction technique is applied and FFT block is used to get the frequency domain data set from the time domain values. The signal in frequency domain represents the data symbols which were mapped to M-PSK or M-QAM. After parallel to serial conversion, these symbols are used to estimate the original data values [16].

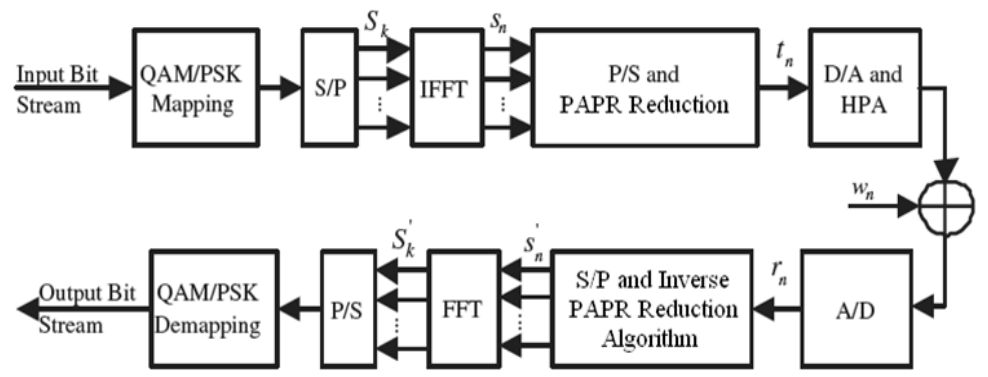

Fig1. OFDM system with PAPR reduction block

\section{Clipping And Differential Scaling}

In this section a new technique called Clipping and Differential Scaling. The probability distribution of amplitudes of the OFDM signal follows Rayleigh distribution [9] and thus the probability of high peaks is very less. An upper threshold above which the signal amplitudes do not contribute much to the signal is determined as follows.

8.1Clipping; Using simulations, we have determined BER for the modified signals along with PAPR. We select the clipping threshold at which the BER is degraded from $1.5 \times 10-3$ to $3.5 \times 10-3$ at SNR of $10 \mathrm{~dB}$ and the amplitudes above this clipping threshold are clipped. Instead of clipping the signal further to reduce the PAPR, we consider a reversible process - Differential Scaling which would reduce the PAPR but not deteriorate the BER. Since different ranges of amplitudes of the signal are scaled in a different manner, it is called Differential Scaling. We have considered three types of scaling as described below.

8.2Scale Up: In this method, we scale up the lower amplitudes of the signal by a factor of $\beta$. This leads to increase the average value without affecting the peak values. Therefore, the resulting PAPR reduces. The PAPR reduction function can be defined as

$$
\begin{aligned}
h(x) & =\alpha x p, \text { if } x>\alpha x p \\
& =\beta x, \text { if } x<A \\
& =x, \text { if } A \leq x \leq \alpha x p
\end{aligned}
$$

where $x p$ is the amplitude peak value occurring in an OFDM symbol block, $\alpha$ is the factor deciding the clipping threshold in terms of percentage of the peak value and $\beta$ is the scaling factor for the range $[0, A)$ whose value is greater than one. The values of the parameters used are mentioned at the end of this section. 
8.3Scale Down: In this method, we scale down the higher amplitudes of the signal by a factor of $\gamma$. This leads to decrease the peak value. Although the average value would also fall down, the resulting PAPR reduces. Because the reduction in peak power is greater than the reduction in the average power. The PAPR reduction function can be defined as

$$
\begin{aligned}
h(x) & =\alpha x p, \text { if } x>\alpha x p \\
& =\gamma x, \text { if } B \leq x \leq \alpha x p \\
& =x, \text { if } x<B
\end{aligned}
$$

where $x p$ is the amplitude peak value occurring in an OFDM symbol block, $\alpha$ is the factor deciding the clipping threshold in terms of percentage of the peak value and $\gamma$ is the scaling factor for the range $[\beta, \alpha x p]$ whose value is less than one. The values of the parameters used are mentioned at the end

8.4Scale Up and Down: in this method, we combine both the above-mentioned approaches i.e. up-scaling and down-scaling. This method exploits the advantages of both the methods. Hence, a PAPR can be reduced considerably. The PAPR reduction function can be defined as

$$
\begin{aligned}
h(x) & =\alpha x p, \text { if } x>\alpha x p \\
& =\gamma x, \text { if } B \leq x \leq \alpha x p \\
& =\beta x, \text { if } x<A \\
& =x, \text { if } A \leq x \leq B
\end{aligned}
$$

Where $x p$ is the amplitude peak value occurring in an OFDM symbol block, $\alpha$ is the factor deciding the clipping threshold in terms of percentage of the peak value. $\beta$ is the scaling factor for the range $[0, A)$ and $\gamma$ is the scaling factor for the range $[B, \alpha x p]$.

\section{Simulation Results} follows

In this paper we have used MATLAB tool to implement this technique and obtained results are as

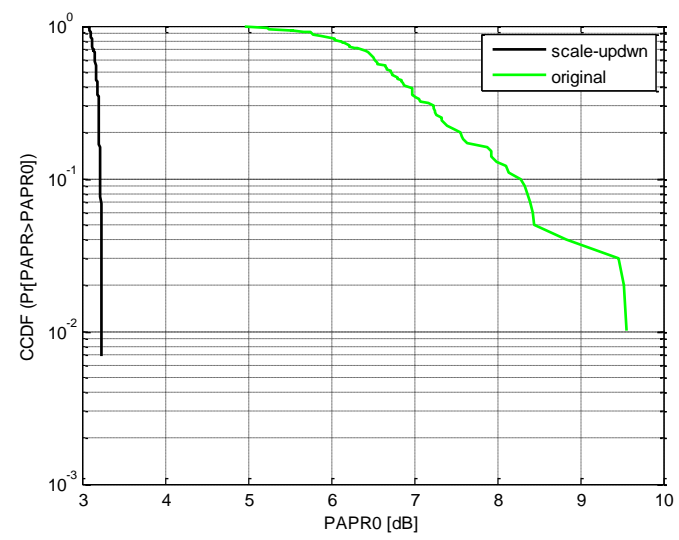

Fig2: A comparision of original signal and scale-updown signal

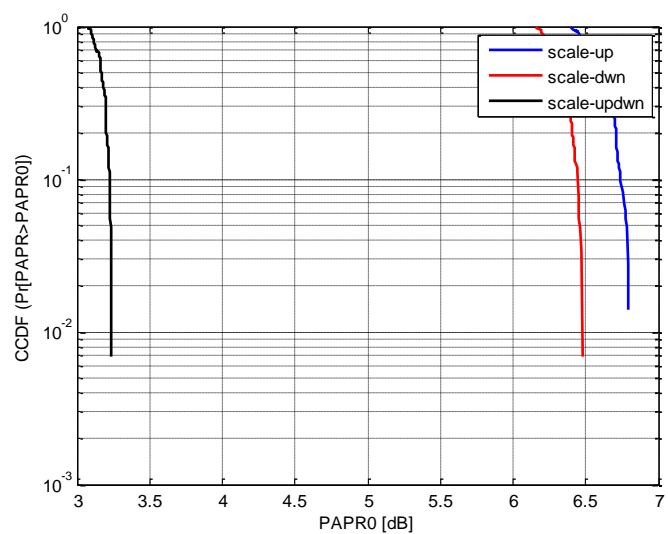

Fig3: A comparision of scale-up,scale-down,scale-updown 


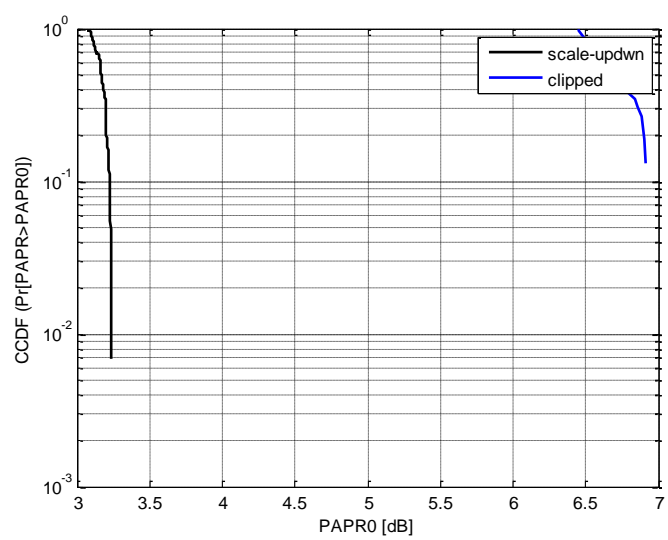

Fig4: A comparision of clipped signal and scale-updown

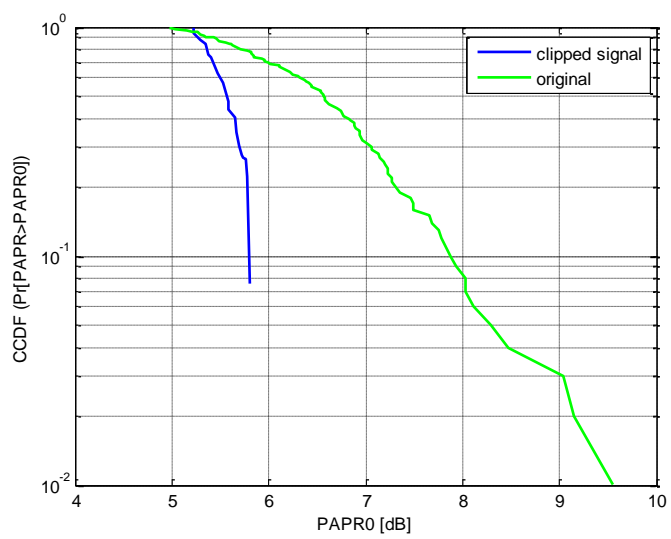

Fig5: A comparision of original signal and clipped signal

\section{Conclusion}

In this paper, we have used a simple approach based on Clipping and Differential Scaling to reduce the PAPR of OFDM signals. We have used Clipping along with three different scaling methods, namely up scaling, down scaling and up-down scaling. Using simulations, we obtained the values of threshold for clipping and parameters for scaling with a view to reduce PAPR without degradation in BER. We have presented the PAPR and BER performance for all the techniques considered. The proposed up-down scaling technique is able to achieve PAPR reduction of the order of $8.5 \mathrm{~dB}$ from $12 \mathrm{~dB}$ PAPR initially. The proposed technique is able to achieve a PAPR of $3.5 \mathrm{~dB}$ while maintaining the BER within a margin of 3 times the BER value at the performance bound at an SNR of $10 \mathrm{~dB}$.

\section{Journal Papers:}

\section{References}

[1] R.W Chang, “Orthogonal Frequency Division Multiplexing,” U.S Patent 3388455,Jan 6, 1970, Filed Nov.4.1966.

[2] T. Hwang, C. Yang, G. Wu, S. Li, and G. Y. Lee, "OFDM and its wireless application: A survey," IEEE Trans. Veh. Technol., vol. 58, no. 4, pp. 1673-1694, May 2009.

[3] A. Jha, Md. Tariq Hasan, and S.M., "Reduction of PAPR in OFDM: A novel approach based on clipping and amplification," IEEE Computer Society, 2009.

[4] T. Jiang and G. Zhu, "Nonlinear companding transform for reducing peak-to-average power ratio of ofdm signals," IEEE Transactions on Broadcasting, Sept. 2004.

[5] B. S. Krongold and D. L. Jones, "An active-set approach for OFDM PAR reduction via tone reservation," IEEE Trans. Signal Process., vol. 52, no. 2, pp. 495-509, Feb. 2004.

[6] L. Wang and C. Tellambura, "Analysis of clipping noise and tonereservation algorithms for peak reduction in OFDM systems," IEEE Trans. Veh. Technol., vol. 57, no. 3, pp. 1675-1694, May 2008

[7] H. Gacanin and F. Adachi, "Selective mapping with Symbol Re-mapping for OFDM/TDM using MMSE-FDE," IEEE VTC, 2008.

[8] Y. Handali, I. Nizan and D. Wulich, "On channel capacity of OFDM with SLM method for PAPR reduction," IEEE 24th Convention of Electrical and Electronics Engineers in Israel, 2006.

[11] J. A. Davis and J. Jedwab, "Peak-to-mean power control in OFDM, Golay complementary sequences, and Reed-Muller codes," IEEE Trans.Inf. Theory, vol. 45, no. 7, pp. 2397-2417, Nov. 1999.

[12] T. Jiang and X. Li, "Using fountain codes to control the peak-to-average power ratio of OFDM signals," IEEE Trans. Veh. Technol., vol. 59, no. 8, pp. 3779-3785, Oct. 2010. 
[13] K. Bae, J. G. Andrews, and E. J. Powers, "Adaptive active constellation extension algorithm for peak-to-average ratio reduction in OFDM," IEEE Commun. Lett., vol. 14, no. 1, pp. 39-41, Jan. 2010.

[14] E. Costa, M. Midro, and S. Pupolin, "Impact of amplifier nonlinearities on OFDM transmission system performance," IEEE Commun. Lett., vol. 3,pp. 37-39, Feb. 1999.

[15] Dae-Woon Lim, Seok-Joong Heo, and Jong-Seon No "An Overview of Peak-to-Average Power Ratio Reduction Schemes for OFDM Signals", journal of communications and networks, vol. 11, no. 3, june 2009, (Invited Paper)

[16] PAPR Reduction in OFDM Systems: A Simple Approach Based on Clipping and Differential Scaling, Aditya Aggarwal, Prachi Kapadia,Y. N. Trivedi, 978-1-4244-9190-2/11/\$26.00 @2011 IEEE

\section{BOOKS:}

[9] Andreas F. Molisch, Wireless Communications, John Wiley, 2002.

[10] D. Brillinger, Time Series Data Analysis and Theory. Philadelphia, PA: SIAM, 2001 